\title{
Design and Implementation of Intelligent Network Configuration Tool
}

\author{
Sayed Sayeed Ahmad* and M. A. Anwar
}

Al Ghurair University, Dubai, UAE; saeed.ks@gmail.com, sahmed@agu.ac.ae, anwar@agu.ac.ae

\begin{abstract}
Objectives: Manage the network related services to reduce the man power to meet business requirements in a competitive environment. Methods: A combination of hardware and software system is developed using System Development Life Cycle (SDLC) to administer any active individual components of a network using TC/IP protocol and Telnet 25 as application layer ${ }^{11}$. The system has advantage over the existing conventional methods to manage the network components without physical accessing the individual component or components in bulk. Findings: The tool presented in this study can be considered as the core of future network management system and can be integrated with other in house developed systems. The system facilitates a network engineer to utilize such tool as it is compatible with any manageable network device that supports tcp/ip8and telnet session10. An engineer while sitting on his seat can manage network devises without physically accessing the devices contrary to the existing method that require configuring a device at the location of the device. The tool is customizable for adding more functionality, such as readymade buttons with pre-programmed scripts to enhance the scalability of the tool. The gui presents all of the functionalities of the system which could be invoked through different buttons such as backup etc. Application/Improvements: The system can be applied to manage the network in bulk remotely and improved the efficiency in network management industry. It could be further improved by making intelligent by integrating with the historical knowledge.
\end{abstract}

Keywords: Automation, Bandwidth, Configuration Tool, Intelligent Network, NetCat

\section{Introduction}

A network management tool - hardware or software facilitates an Information Technology professional to manage the individual components of any network within a larger network administration structure. The bandwidth management that focuses on network performance is one of the important issues today in computer engineering applications and systems mainly in Network Management $\frac{1,7.7}{}$. By controlling the amount of bandwidth to an application or user, the network administrator can prevent a small number of applications or users to consume all available bandwidth ${ }^{2,8}$. The tool may assist to identify the devices that are part of network, monitor the working of a network component at the device level, and observe whether the performance of the devices is according to the capacity plans, continuously monitoring the indicators of performance i.e. bandwidth usage, loss of packets, and generating alerts for proposed configuration for a particular network situation through emails, phone calls, SMSs, and paging for a network administrator ${ }^{12}$.

The Internet applications have become a very bandwidth demanding in recent years and further increase is expected in future. The diverse and rising demand for broadband in hotel industry is forcing up the need for bandwidth at a fasterspeed ${ }^{2}$. A study was conducted in 2010 for CISCO which is a supplier of networking infrastructure and found that the growth rate was approximately $4.45 \%$ per month 2 . This is equivalent to an annual CAGR of around $60 \%$. The multimedia application are creating a challenge for the internet operators to provide more internet bandwidth and faster downloads for the large files such as movies, and the online gaming is also putting a big load on the backbone of the Internet. The hotel or restaurant management can make it possible to provide the high-speed access of the Internet to

*Author for correspondence 
their members by increasing the available capacity to overcome the spike of use without spending more on a larger broadband ${ }^{2,10}$. It has become a challenging task for the operators to upgrade the internet speed individually for their subscribers on demand as it is a time consuming and it requires a large number of man power.

The Network Configuration tools "The NetCat" presented in paper is a combination of hardware and software designed in Visual Basic version 6.0 $0^{1}$ that allow to a technician sitting on his/her desk to send network command to any active components remotely using TCP/ IP $\$ .9$ protocol and using Telnet port 25 as application layer using GUI. "The NetCat" facilitates in handling the day to day operations of an Internet Service provider such as minimizing the operational expenses by reducing the cost of the man power, ensure better service delivery by carrying a massive change on a timely manner, etc. "The NetCat" can easily be incorporated with available tools available for managing a network.

The remainder of the paper is organized as follows; in section 2 we present the proposed system logical model, in section 3 the system itself is explained, and in section 4 we conclude our work.

\section{The NetCat System Logical Model}

The system development life cycle (SDLC) is an important process uses during the development of any system $\frac{5,6}{}$. The SDLC consists of four main phases; planning, analysis, design and implementation. The process model is captured using the context level diagrams and data flow diagrams (DFD) in the analysis phase. The DFDs are mostly used to present the system requirements during the analysis phase. A context level DFD is used to show the functionality of a system as a whole at highest level and it does not contain any data stores. The context level DFD contains only one process and shows the entire data flowing either into or out of the process. The DFD actually presents how external entities will interact with the systems ${ }^{3}$. The context level DFD of the proposed system is shown in Figure 1. There are three types of users; ISP User I, ISP User II and ISP User III, who will interact with the NetCat configuration system for different purposes.

The purpose of DFD at level show an overview of the entire system and these diagrams present the functionaly of the system in more detail. The major processes are divided into sub-processes and it also icludes data stores that are used for storing the data by the major processes. The level I DFD of the systems is shown in Figure 2. The users of the system can enter new policy, new IP list, and new script to be executed for the required changes. The relevant sub-processes do the required task, send the confirmation message and update the logs.

\section{The NetCat System}

The application starts with a splash screen with a brief description of the tool capabilities and with a warning for the user to make sure that user knows the actual functions of the proposed tool. The second main interface (main menu) as shown in Figure 3 appears if user is successfully logged in the system. This interface presents all of the functionalities of the system which could be invoked through different buttons. The flow of the steps undertaken to upgrade the Internet speed is shown as a flowchart in Figure 4.

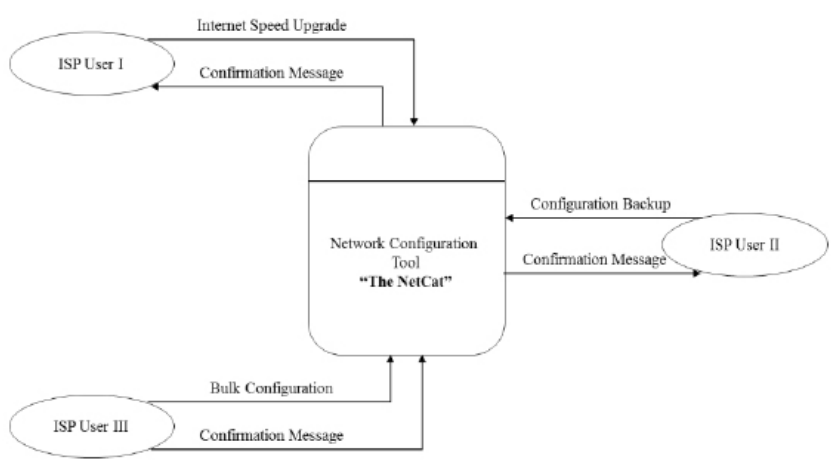

Figure 1. The NetCat context level DFD.

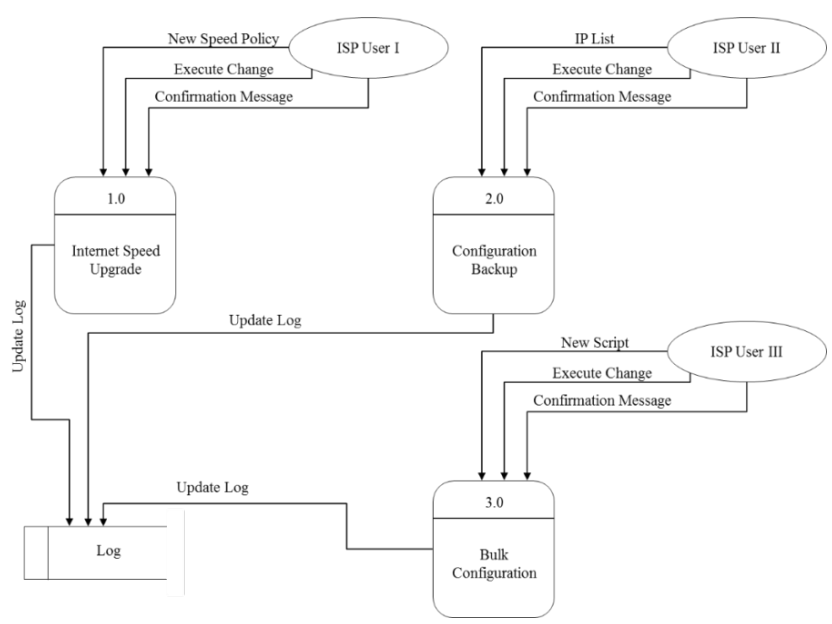

Figure 2. The NetCat context level I DFD. 


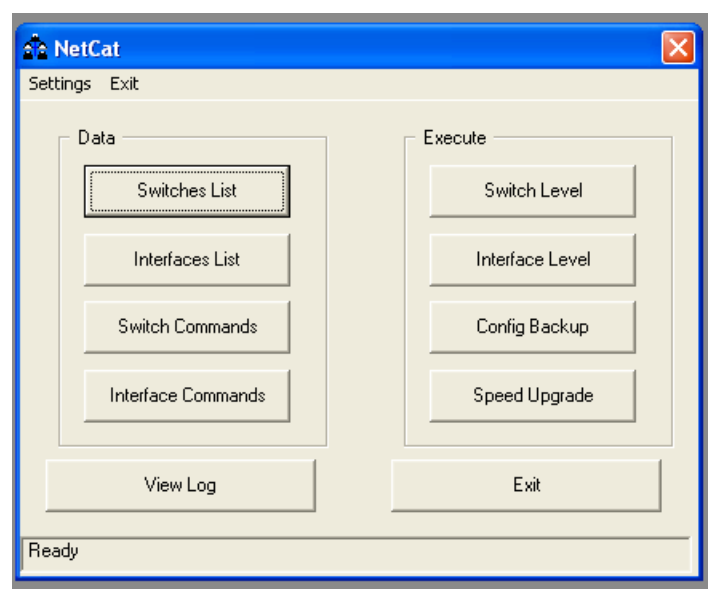

Figure 3. The NetCat Main GUI.

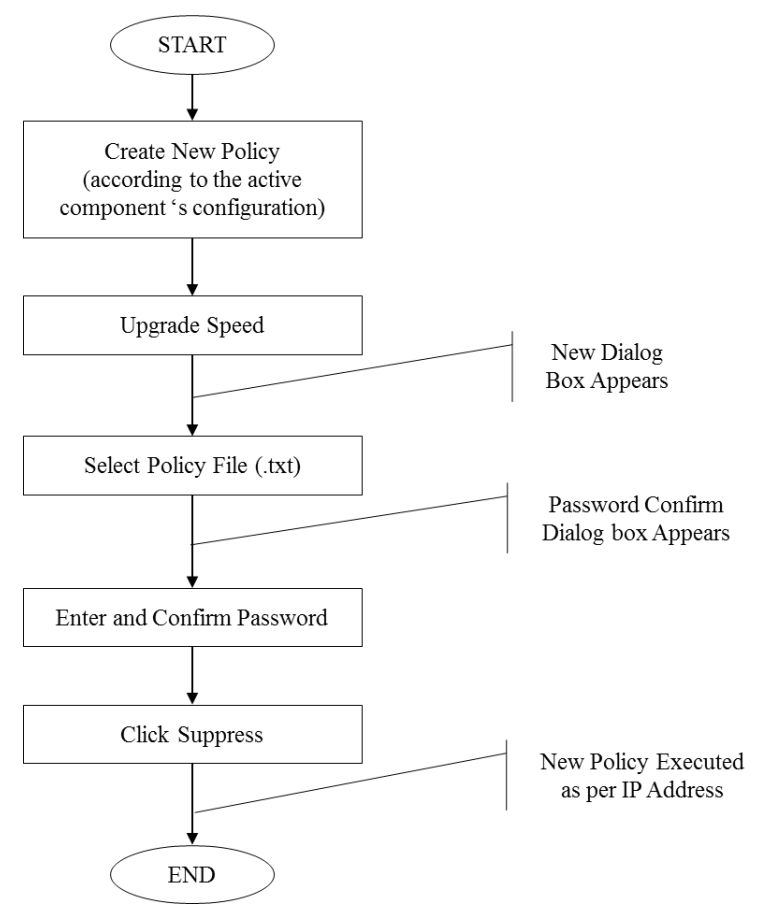

Figure 4. The NetCat context level DFD.

The user can perform an automatic backup of the running configuration of all the devices using the "Configuration Backup" button as follows:

1. logon to the "The NetCat" system

2. press "Configuration Backup" button

3. application will then TELNET to the entire network devices according to the pre-stored list of the devices IP addresses

4. application will perform a network command to copy the running configuration and save it under a prespecified folder namely "Configuration Backup"
5. a message box will popup notifying the user that the backup is completed

When the user is done with whatever operation (speed upgrade, bulk configuration or configuration backup) and he is in need to check the status of the previous transactions. The logs are also maintained for this purpose. To see the logs, the user will:

1. Open the "The NetCat" application

2. Click on logs

3. Then check the correspondence file according to the date of the activity

4. Save them or take them on a notepad.

In the following paragraphs, we present some of the GUIs snapshots of the systems. When a user clicks on Switches List button a dialog box as shown in Figure 5 appears asking the user to add the new IP list that the "The NetCat" will access to send the configuration.

To amend or change the configuration of the interfaces that will apply to all switches click on the "Interface List" and a dialog box as shown in Figure 6 will appear to complete this task.

The Interface Level is used to store and save the commands which are needed to be applied on the interfaces such as the VLAN ID, the description or the new

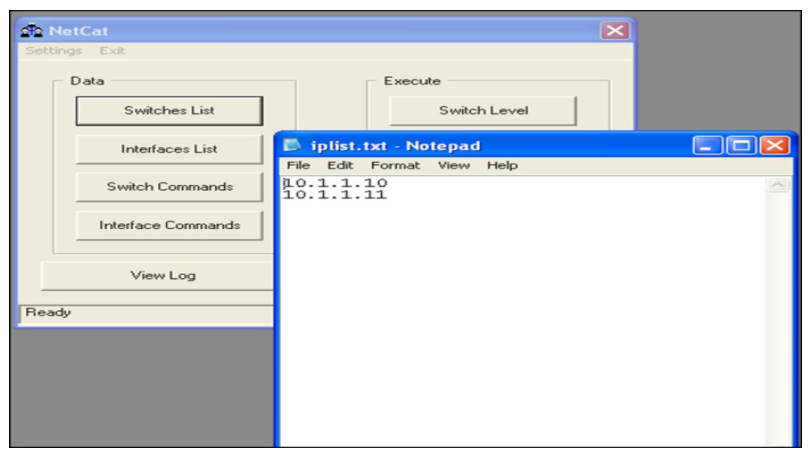

Figure 5. New IP add dialog box.

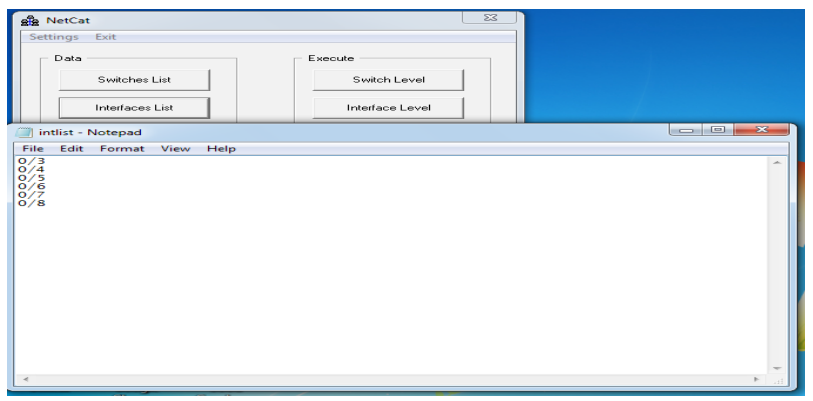

Figure 6. Select Interface dialog box. 


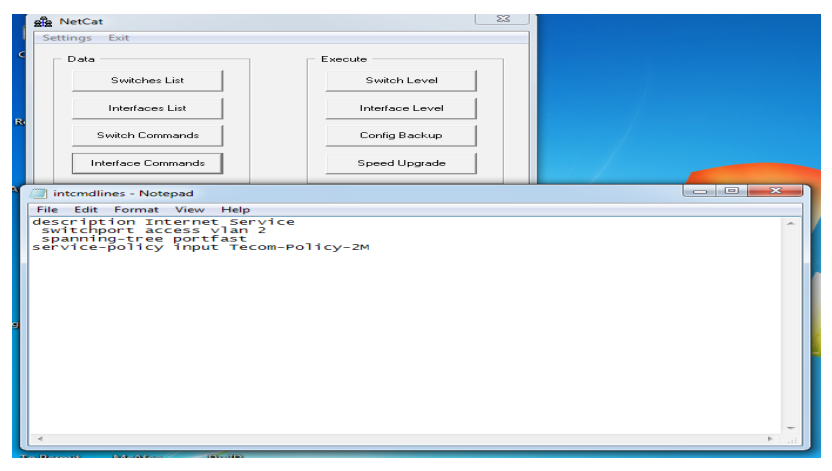

Figure 7. Interface level dialog box.

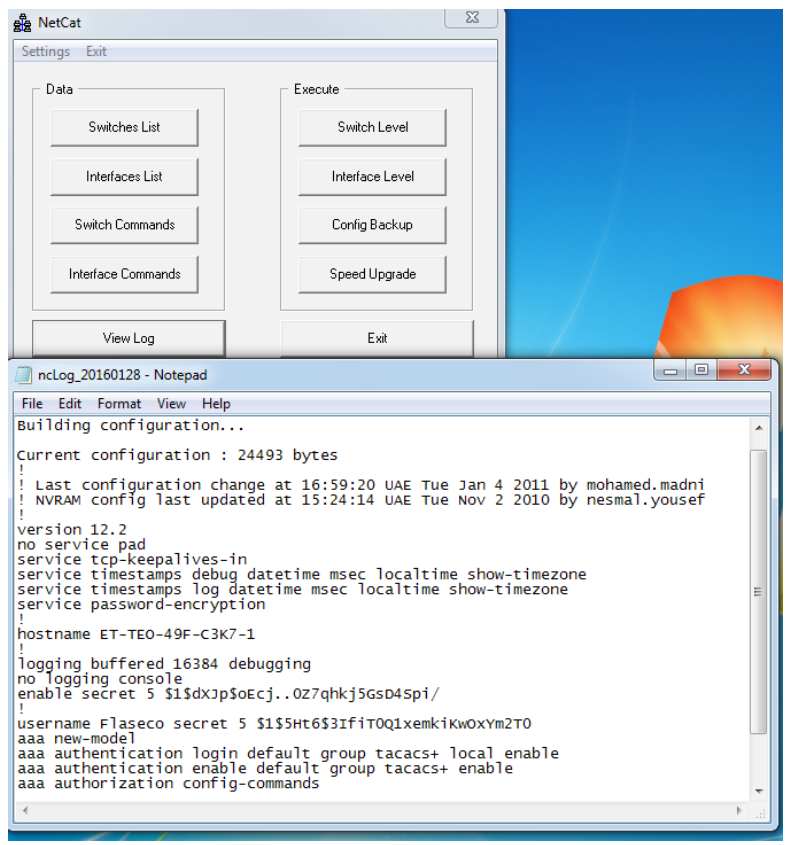

Figure 8. View logs GUI.

speed if required. The same will applied for all interfaces by one click and the tool will telnet to all devices and do the needful. This is shown in Figure 7.

The system also provides functionality to view and verify the logs by clicking on the View Logs button as shown in Figure 8. It is very useful interface which can show you if the tool was unable to reach any switch from the list due to network connectivity issue.

\section{Conclusion}

This tool can be considered as the core of future network management system. With continues work and development, integrating it with other in house developed systems it will make a powerful tool.
Overall, it is the easy for the network engineer to utilize such tool as it is compatible with any manageable network device that supports TCP/IP and telnet session. The tool is customizable for adding more functionality, such as readymade buttons with pre-programmed scripts to enhance the scalability of the tool.

\section{Acknowledgements}

The authors wish to acknowledge the support of Al Ghurair University for its support to develop and implement this system.

\section{References}

1. Visual Basic 6.0 Resource Center. Available from: https:// msdn.microsoft.com/en-us/vstudio/ms788229.aspx. Date accessed: 01/11/2016

2. Hospitality matters - Bandwidth on-demand: Enhancing the guest experience with affordable high-speed Internet. Available from: http://www.du.ae/Documents/Du-WhitePaper-Hospitality-matters-Bandwidth-on-demand-web. pdf. Date accessed: 01/11/2016

3. Context LevelDFS's \& Level 1.DFD's. Available from:https:// eternalsunshineoftheismind.wordpress.com/2013/03/05/ context-level-dfds-level-1-dfds/comment-page-1/Date accessed: 01/11/2016

4. Govindarajan J, Vibhurani N, Kousalya G. An Analysis on TCP Packet Reordering Problem in Mobile Ad-Hoc Network. Indian Journal of Science and Technology. 2015 Jul; 8(16). Doi no:10.17485/ijst/2015/v8i16/62902

5. McMurtrey M. A Case Study of the Application of the Systems Development Life Cycle (SDLC) in 21st Century Health Care: Something Old, Something New? The Journal of the Southern Association for Information Systems (JSAIS). 2013; 1(1). Doi: 10.3998/jsais.11880084.0001.103.

6. Balaji S, Murugaiyan M. Waterfall vs. V-Model vs. Agile: A comparative study on SDLC. International Journal of Information Technology and Business Management. 2012; 2(1):26-30.

7. Kassim M, Kassim H. An Analysis on Bandwidth Utilization and Traffic Pattern for Network Security Management. Journal of International Proceedings on Computer Science and Information Technology. 2011 Oct; 13:51-6.

8. Balameena N, Muralidharan D. Automation of wireless Telecom Node Commands of Network Management System. Indian Journal of Science and Technology. 2016 Aug; 9(29). Doi: 10.17485/ijst/2016/v9i29/93212.

9. Rajkumar K, Swaminathan P. Combining TCP and UDP for Secure Data Transfer. Indian Journal of Science and 
Technology. 2015 May; 8(S9). Doi: 10.17485/ijst/2015/ v8iS9/65569.

10. Anbar M, Ramadass S, Manickam S, Al-Wardi A. Connection Failure Message-based Approach for Detecting Sequential and Random Tcp Scanning. Indian Journal of Science and Technology. 2014 Jan; 7(5). Doi: 10.17485/ ijst/2014/v7i5/49734.

11. Sindhu Raj K, Thanudas B, Radhika N, Smitha VS. SatelliteTCP: A Flow Control Algorithm for Satellite Network.
Indian Journal of Science and Technology. 2015 Aug; 8(17). Doi: 10.17485/ijst/2015/v8i17/65032.

12. Govindarajan J, Vibhurani N, Kousalya G. An Analysis on TCP Packet Reordering Problem in Mobile Ad-Hoc Network. Indian Journal of Science and Technology. 2015 Jul; 8(16). Doi:10.17485/ijst/2015/v8i16/62902. 